\title{
NEW ANTAGONIST AGENTS OF NEUROPEPTIDE Y RECEPTORS
}

Ignacio Aldana , Isabel Rivero, Argimiro Rivero, Patricio Huenchuñir, Carmen Frigola, Maria Luisa Alonso e Antonio Monge Department of Pharmaceutical Chemistry - CIFA - University of Navarra - Pamplona - Spain

D. H. Caignard and P. Renard

ADIR - Courbevoie Cedex - France

Recebido em 2/9/99; aceito em 20/4/00

In the CNS, NPY has been implicated in obesity and feeding, endocrine function and metabolism. Potent and selective rNPY antagonists will be able to probe the merits of this approach for the treatment of obesity. We report the synthesis and preliminary evaluation of some hydrazide derivatives as antagonists of rNPY.

Keywords: obesity; energy metabolism; weight reduction; neuropeptide Y.

\section{INTRODUCTION}

The Neuropeptide Y (NPY) is a peptide of 36 amino acids, similar to the peptide YY (PYY) and to the pancreatic polypeptides (PP) ${ }^{1}$. At first, separated from the pig brain, the NPY is well-distributed in the mammals at the level of the central and peripheric nervous system. This neurotransmitter is present in strong concentrations in the nervous fiber of the brain, but equally as strong in the heart, the sympathetic glands, the blood vessels and the gastrointestinal tract. It is responsable for diverse phyisiological effects that exert themselves by way of specific receptors $(\mathrm{Y})$. These $\mathrm{Y}$ receptors form a heterogeneous group and six subtypes have been identified up to now: Y1 to $\mathrm{Y}^{2}$. The NPY is involved in the control of appetite, strongly stimulating the intake of food, or performing a regulatory role on the HPA (hypothalamic-pituitary-adrenal) axis ${ }^{3}$. It also presents anxiolytic and sedative properties that induce an increase in the blood pressure and affects the circadian rhythm ${ }^{4}$.

The search for agonists and antagonists of NPY could yield compounds which could be used in the treatment of pathologies related to food behavior or energy equilibrium disorders, such as diabetes, obesity, bulimia and anorexia. Also in the treatment of arterial hypertension, anxiety, depressions, epilepsia and sleeping disorders.

The orexigenic effects of NPY could be mediated by it receptor NPY1 subtype ${ }^{5}$, which makes it possible to consider that is involved in the stimulating effect of the appetite of the neuropeptide Y. Recently, ligands of this receptor have been described. The first compound of nonpeptide structure with potent antagonist activity of the receptor NPY1, is BIBP 3226 (Fig.1), which was designed using the region C-terminal of the $\mathrm{NPY}^{6}$. Recent studies appear to confirm an important implication of the receptor NPY5 subtype in the control of food ingestion ${ }^{7}$. The first Y5-selective antagonists appeared in the patent literature in mid- $1997^{8}$. These compounds have something in common: an amino acid, phenylalanine (SR 120819A) and arginine (BIBP 3226) respectively, in the center part of the molecule. Also, the amino and carboxyl groups from the amino acid remain funtionalized in the form of amide. Likewise, the first selective NPY5 antagonists are appearing. One such example is JCF 104 (2-(naphtalen-1-yl)-3-phenylpropane-1,2diamine $)^{9}$ (Fig. 1), which carries a rest of 2-amino-3phenylpropanamine, closely related to the aforementioned systems. Our objective is to search for new compounds that are non-peptidic selective antagonists of the Y5 receptor subtype. This line of research implies the presence of a phenylalanine and/or methyltyrosine rest in the central part of a molecule in which the carboxyl and amine groups of the amino acid are funtionalized as amide and carbamate respectively (Fig. 2).
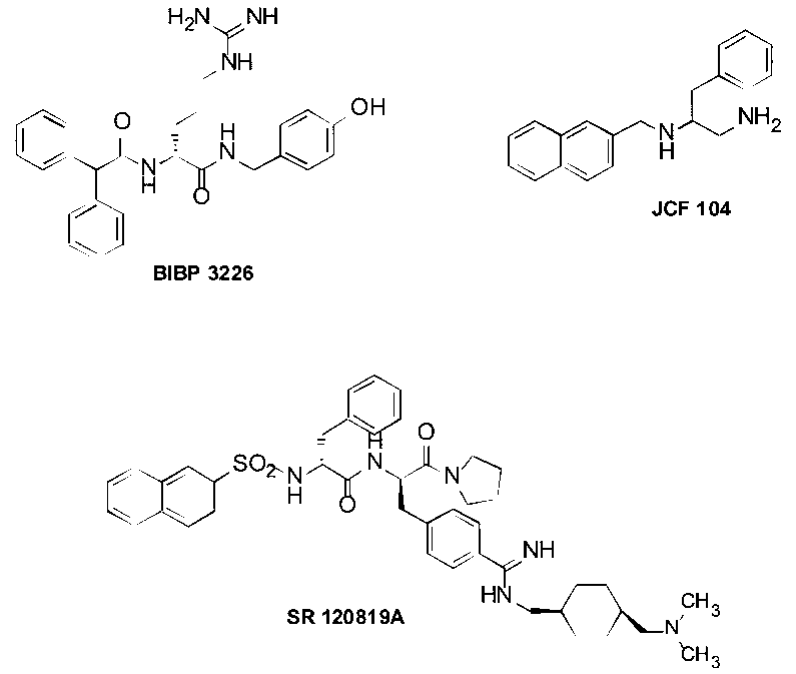

Figure 1. Antagonists of the NPY receptors.<smiles>[R2]NC(=O)[C@H](Cc1ccc([R])cc1)NC(=O)OCc1ccccc1</smiles>

Figure 2. General structure for the synthetized compounds.

In this work, the first experimental results are offered. All the synthetized compounds were evaluated for their antagonist action of NPY receptors. Their action was evaluated on the NPY Y1, Y2, Y5 subtype receptors.

\section{CHEMISTRY}

Chemicals, methods and apparatus

General laboratory chemicals were purchased from Merck, Sigma, Janssen, and Scharlau. L-phenylalanine, Lmethyltyrosine, HOBT and EDC was purchased from Fluka. 
All the new compounds were characterized by elemental analysis, IR and ${ }^{1} \mathrm{H}$ NMR. The IR spectra are recorded on a Perkin-Elmer FT 681 using $\mathrm{KBr}$ pellets. The ${ }^{1} \mathrm{H}$ NMR spectra are obtained on a Bruker Model AC-200E (200 MHz) with $\mathrm{Me}_{4} \mathrm{Si}$ as the internal standard, and at a concentration of approximately $0.1 \mathrm{~g} / \mathrm{mL}$. The mass spectra were obtained on a Hewlett-Packard HP-5890 (GC/HPLC/DIP) instrument. All spectra were consistent with assigned structures. Thin layer chromatography was performed on aluminum sheets precoated with silica gel (HF 254, Merck). The developed chromatograms were viewed under UV light or iodine revelation. Melting points were determined on a Mettler FP82 hot stage apparatus equipped with a FP800/FP80 processor and an Olympus 8091 microscope provided with a video system and were uncorrected. Elemental analyses of vaccuum-dried samples were obtained on a Carlo Erba 1106 elemental analyzer (over $\mathrm{P}_{2} \mathrm{O}_{5}$ at 1-2 $\mathrm{mmHg}, 24 \mathrm{~h}$ at $60-80^{\circ} \mathrm{C}$ ). Results are within $0.4 \%$ of theoretical values unless otherwise indicated.

\section{Synthesis of the compounds}

General procedure for the preparation of 3-H and 3-(4methoxyphenyl)propionic acid derivatives. a-b

The appropiate amino acid was dissolved in $\mathrm{NaOH} 4 \mathrm{M}$. The solution was cooled to $0^{\circ} \mathrm{C}$ in an ice bath. A solution of the benzyl chloroformate (equimolar amount) in dry $\mathrm{CH}_{2} \mathrm{Cl}_{2}$ and $\mathrm{NaOH} 4 \mathrm{M}$ were added alternately and portionwise, with stirring and cooling, during 30 minutes. The mixture was allowed to warm with intermitent shaking for $1 \mathrm{~h}$. The mixture was extracted with ethyl ether $\left(2 \_15 \mathrm{~mL}\right)$. The aqueous layer was acidified with diluted $\mathrm{HCl}(1: 1)$ to $\mathrm{pH} 2$. The solid was filtered and washed with ethyl ether $(3 \times 10 \mathrm{~mL})$ in order to obtain the product as a solid.

(S)-2-Benzyloxycarbonylamino-3-phenylpropanoic acid (a) From L-Phe $(13.20 \mathrm{~g}, 80.00 \mathrm{mmol})$ and benzyl chloroformate (11.25 g, $80.00 \mathrm{mmol})$. A white solid $(20.87 \mathrm{~g}, 87 \%) \mathrm{mp} \mathrm{84.9-}$ 85.4: IR $\left(\mathrm{KBr}, \mathrm{cm}^{-1}\right) 3329(\mathrm{~s}, \mathrm{~N}-\mathrm{H}), 3033(\mathrm{~m}$, aromatic C-H), 2942 (m, aliphatic C-H), 1696 (vs, carbamate and carboxylic acid $\mathrm{C}=\mathrm{O}$ ), 748 and 697 (m, aromatic monosubstitution); ${ }^{1} \mathrm{H}$ NMR $\left(\mathrm{CDCl}_{3},{ }^{\delta}\right)$ 2.74-3.10 (d, d, d, d, 2H, CH $5.3,6.2,6.4 \mathrm{~Hz}$ ), 4.06-4.17 (dd, $1 \mathrm{H}, \mathrm{CH}, \mathrm{J}=6.0 / 13.8 \mathrm{~Hz}), 4.93$ $\left(\mathrm{s}, 2 \mathrm{H}, \mathrm{CH}_{2}-\mathrm{O}\right), 6.42(\mathrm{~d}, 1 \mathrm{H}, \mathrm{NH}, \mathrm{J}=7.7 \mathrm{~Hz}), 7.05-7.35(\mathrm{~m}$, $10 \mathrm{H}, \mathrm{C}_{6} \mathbf{H}_{5}-\mathrm{CH}_{2}-\mathrm{CH}$ and $\mathrm{C}_{6} \mathbf{H}_{5}-\mathrm{CH}_{2}-\mathrm{O}$ ), 10.18 (ws, $1 \mathrm{H}, \mathrm{OH}$ ); MS m/z 107.05 (10.56), 91.05 (100), 76.95 (12.00); Anal. $\left(\mathrm{C}_{17} \mathrm{H}_{17} \mathrm{NO}_{4}\right) \mathrm{C}, \mathrm{H}, \mathrm{N}$.

(S)-2-Benzyloxycarbonylamino-3-(4-methoxyphenyl)propanoic acid $(\mathbf{b})$

From O-Methyl-L-Tyr (0.50 g, $2.50 \mathrm{mmol})$ and benzyl chloroformate $(0.36 \mathrm{~mL}, 2.56 \mathrm{mmol})$. A white solid $(0.65 \mathrm{~g}$, 77\%) mp 111.1-113.4: IR (KBr, cm $\left.{ }^{-1}\right) 3305$ (s, N-H), 3033 (m, aromatic C-H), 2956 (m, aliphatic C-H), 1696 (vs, carbamate and carboxylic acid $\mathrm{C}=\mathrm{O}$ ), 746 and 695 (aromatic monosubstitution); ${ }^{1} \mathrm{H}-\mathrm{NMR}\left(\mathrm{CDCl}_{3}, \delta\right) 3.01-3.22$ (d, t, d, $\left.2 \mathrm{H}, \mathrm{CH}_{2}-\mathrm{CH}, \mathrm{J}=5.9,6.0,5.5 \mathrm{~Hz}\right), 3.78\left(\mathrm{~s}, 3 \mathrm{H}, \mathrm{OCH}_{3}\right), 4.62-$ $4.71(\mathrm{dd}, 1 \mathrm{H}, \mathrm{CH}, \mathrm{J}=5.9 / 13.5 \mathrm{~Hz}), 5.11\left(\mathrm{~s}, 2 \mathrm{H}, \mathbf{C H}_{2}-\mathrm{O}\right), 5.21$ $(\mathrm{d}, 1 \mathrm{H}, \mathrm{NH}, \mathrm{J}=8.1 \mathrm{~Hz}), 6.82\left(\mathrm{~d}, 2 \mathrm{H}, \mathbf{H}_{2}\right.$ and $\mathbf{H}_{6}$ of $\mathrm{C}_{6} \mathrm{H}_{4}-$ $\left.\mathrm{OCH}_{3}, \mathrm{~J}=8.5 \mathrm{~Hz}\right), 7.07\left(\mathrm{~d}, 2 \mathrm{H}, \mathbf{H}_{3}\right.$ and $\mathbf{H}_{5}$ of $\mathrm{C}_{6} \mathrm{H}_{4}-\mathrm{OCH}_{3}$, $\mathrm{J}=8.5 \mathrm{~Hz}), 7.35\left(\mathrm{~s}, 5 \mathrm{H}, \mathrm{C}_{6} \mathbf{H}_{5}-\mathrm{CH}_{2}\right)$; MS m/z 329.05 (1.50, $\left.\mathrm{M}^{+\cdot}\right), 121.15$ (100), 91.05 (12.09), 77.05 (15.22); Anal. $\left(\mathrm{C}_{18} \mathrm{H}_{19} \mathrm{NO}_{5}\right) \mathrm{C}, \mathrm{H}, \mathrm{N}$.

General procedure for the preparation of final compounds (1-13) A mixture of the appropiate propanoic acid derivative and HOBT in dry $\mathrm{CH}_{2} \mathrm{Cl}_{2}$ at $0^{\circ} \mathrm{C}$, under $\mathrm{N}_{2}$, was treated with EDC. After $1 \mathrm{~h}$ at $0^{\circ} \mathrm{C}$, a solution of the appropiate hydrazide or hydrazine, in dry $\mathrm{CH}_{2} \mathrm{Cl}_{2}$ was added (equimolar amount of HOBT, EDC, and hydrazide or hydrazine was used). The reaction was stirred at $0^{\circ} \mathrm{C}$ for $1 \mathrm{~h}$ and then at room temperature for $24 \mathrm{~h}$. The solvent was evaporated. The residue was redissolved in ethyl acetate or $\mathrm{CH}_{2} \mathrm{Cl}_{2}$ and washed with water $(3 \times 20 \mathrm{~mL})$. The organic phase was dried over $\mathrm{Na}_{2} \mathrm{SO}_{4}$ and evaporated under reduced pressure. The obtained residue was washed with ethyl ether in order to obtain the product as a solid.

(S)-2-Benzyloxycarbonylamino-3-phenyl-N'-phenylpropionohydrazide (1)

From (a) (1.00 g, $3.34 \mathrm{mmol})$, HOBT (0.58 g, $3.81 \mathrm{mmol})$, EDC $(0.73 \mathrm{~g}, 3.81 \mathrm{mmol})$ and phenylhydrazine $(0.37 \mathrm{~mL}, 3.81$ $\mathrm{mmol})$. A beige solid $(0.53 \mathrm{~g}, 38 \%) \mathrm{mp} 175.0-177.1$ : IR $(\mathrm{KBr}$, $\left.\mathrm{cm}^{-1}\right) 3297$ and $3244(\mathrm{~m}, \mathrm{~N}-\mathrm{H}), 3032$ (w, aromatic C-H), 2920 (w, aliphatic C-H), 1688 (s, carbamate $\mathrm{C}=\mathrm{O}$ ), 1656 (s, hydrazide $\mathrm{C}=\mathrm{O}), 746$ and $696\left(\mathrm{~m}\right.$, aromatic monosubstitution); ${ }^{1} \mathrm{H}$ NMR $\left(\mathrm{CDCl}_{3},{ }^{\delta}\right) 3.07\left(\mathrm{~d}, 2 \mathrm{H}, \mathrm{CH}_{2}-\mathrm{CH}, \mathrm{J}=7.3 \mathrm{~Hz}\right), 4.45-4.60$ (dd, 1H, CH, J=7.4/15.1 Hz), 5.06 (s, 2H, Ph-CH - -O), 5.49 (d, $1 \mathrm{H}, \mathrm{NH}-\mathrm{CH}, \mathrm{J}=8.5 \mathrm{~Hz}), 5.97(\mathrm{~d}, 1 \mathrm{H}, \mathrm{NH}-\mathrm{NH}-\mathrm{Ph}, \mathrm{J}=3.6 \mathrm{~Hz})$, $6.50\left(\mathrm{~d}, 2 \mathrm{H}, \mathbf{H}_{2}\right.$ and $\mathbf{H}_{6}$ of $\left.\mathrm{C}_{6} \mathrm{H}_{5}-\mathrm{NH}, \mathrm{J}=7.9 \mathrm{~Hz}\right), 6.85(\mathrm{t}, 1 \mathrm{H}$, $\mathbf{H}_{4}$ of $\left.\mathrm{C}_{6} \mathrm{H}_{5}-\mathrm{NH}, \mathrm{J}=7.3 \mathrm{~Hz}\right), 7.07-7.35\left(\mathrm{~m}, 12 \mathrm{H}, \mathrm{C}_{6} \mathbf{H}_{5}-\mathrm{CH}_{2}-\mathrm{O}\right.$, $\mathrm{C}_{6} \mathbf{H}_{5}-\mathrm{CH}_{2}-\mathrm{CH}$ and $\mathbf{H}_{3}$ and $\mathbf{H}_{5}$ of $\mathrm{C}_{6} \mathrm{H}_{5}-\mathrm{NH}$ ), 8.01 (ws, $1 \mathrm{H}$, CO-NH-NH); MS m/z $389.20\left(2.90, \mathrm{M}^{+*}\right), 107.15$ (51.51), 91.05 (100); Anal. $\left(\mathrm{C}_{23} \mathrm{H}_{23} \mathrm{~N}_{3} \mathrm{O}_{3}\right) \mathrm{C}, \mathrm{H}, \mathrm{N}$.

(S)- N'-Benzoyl-2-benzyloxycarbonylamino-3-phenylpropionohydrazide(2)

From (a) (1.00 g, $3.34 \mathrm{mmol})$, HOBT (0.56 g, $3.68 \mathrm{mmol})$, EDC $(0.71 \mathrm{~g}, 3.68 \mathrm{mmol})$ and phenylhydrazide $(0.50 \mathrm{~g}, 3.68$ $\mathrm{mmol})$. A beige solid $(0.20 \mathrm{~g}, 14 \%) \mathrm{mp} 172.2-174.5$ : IR $(\mathrm{KBr}$, $\left.\mathrm{cm}^{-1}\right) 3285$ and $3205(\mathrm{~m}, \mathrm{~N}-\mathrm{H}), 3030(\mathrm{w}$, aromatic C-H), 1699 (s, carbamate $\mathrm{C}=\mathrm{O}$ ), 1610 ( $\mathrm{s}$, hydrazide $\mathrm{C}=\mathrm{O}$ ), 744 and 696 (m, aromatic monosubstitution); ${ }^{1} \mathrm{H}-\mathrm{NMR}\left(\mathrm{CDCl}_{3},{ }^{\delta}\right) 2.97-3.24$ (d, d, d, d, 2H, CH $\mathbf{H}_{2}-\mathrm{CH}, \mathrm{J}=8.3,8.2,5.9,6.1 \mathrm{~Hz}$ ), 4.87-5.06 (m, 3H, CH and $\mathbf{C H}_{2}-\mathrm{O}$ ), 5.98 (ws, $1 \mathrm{H}, \mathrm{NH}-\mathrm{CH}$ ), 7.09-7.38 $\left(\mathrm{m}, 12 \mathrm{H}, \mathrm{C}_{6} \mathbf{H}_{5}-\mathrm{CH}_{2}-\mathrm{O}, \mathrm{C}_{6} \mathbf{H}_{5}-\mathrm{CH}_{2}-\mathrm{CH}\right.$ and $\mathbf{H}_{3}$ and $\mathbf{H}_{5}$ of $\mathrm{C}_{6} \mathrm{H}_{5^{-}}$ $\mathrm{CO}), 7.48\left(\mathrm{t}, 1 \mathrm{H}, \mathbf{H}_{4}\right.$ of $\left.\mathrm{C}_{6} \mathrm{H}_{5}-\mathrm{CO}, \mathrm{J}=7.2 \mathrm{~Hz}\right), 7.78\left(\mathrm{~d}, 2 \mathrm{H}, \mathbf{H}_{2}\right.$ and $\mathbf{H}_{6}$ of $\mathrm{C}_{6} \mathrm{H}_{5}-\mathrm{CO}, \mathrm{J}=7.8 \mathrm{~Hz}$ ), 9.52 (ws, $1 \mathrm{H}, \mathrm{CH}-\mathrm{CO}-\mathrm{NH}-$ NH-CO), 9.96 (ws, 1H, CO-NH-NH-CO-Ph); MS $\mathrm{m} / z 282.15$ (14.37), 254.15 (3.91), 105.00 (54.12), 91.05 (100); Anal. $\left(\mathrm{C}_{24} \mathrm{H}_{23} \mathrm{~N}_{3} \mathrm{O}_{4}\right) \mathrm{C}, \mathrm{H}, \mathrm{N}$.

(S)-2-Benzyloxycarbonylamino-N'-(2-indolylcarbonyl)-3phenylpropionohydrazide $(\mathbf{3})$

From (a) $(0.20 \mathrm{~g}, 0.67 \mathrm{mmol})$, HOBT $(0.12 \mathrm{~g}, 0.76 \mathrm{mmol})$, EDC $(0.14 \mathrm{~g}, 0.76 \mathrm{mmol})$ and indol-2-hydrazide $(0.14 \mathrm{~g}, 0.76 \mathrm{mmol})$. A beige solid $(0.15 \mathrm{~g}, 49 \%) \mathrm{mp}$ 176.3-177.5: IR $\left(\mathrm{KBr}, \mathrm{cm}^{-1}\right)$ 3412 (vs, N-H), 3031 (w, aromatic C-H), 2953 (w, aliphatic CH), 1697 (vs, carbamate $\mathrm{C}=\mathrm{O}$ ), 1666 (vs, hydrazide $\mathrm{C}=\mathrm{O}$ ), 745 and 697 (vs, aromatic monosubstitution); ${ }^{1} \mathrm{H}-\mathrm{NMR}$ (DMSO-d $\mathrm{d}_{6}$, б) $2.90\left(\mathrm{t}, 1 \mathrm{H}, \mathrm{CH}_{\mathrm{A}} \mathrm{H}_{\mathrm{B}}-\mathrm{CH}, \mathrm{J}=11.2 \mathrm{~Hz}\right), 3.20\left(\mathrm{~d}, 1 \mathrm{H}, \mathrm{CH}_{\mathrm{A}} \mathbf{H}_{\mathbf{B}^{-}}\right.$ $\mathrm{CH}, \mathrm{J}=11.8 \mathrm{~Hz}), 4.30-4.60(\mathrm{~m}, 1 \mathrm{H}, \mathrm{CH}), 4.98\left(\mathrm{~s}, 2 \mathrm{H}, \mathrm{CH}_{2}-\mathrm{O}\right)$, 6.95-7.55 (m, 13H, $\mathrm{C}_{6} \mathbf{H}_{5}-\mathrm{CH}_{2}-\mathrm{O}, \mathrm{C}_{6} \mathbf{H}_{5}-\mathrm{CH}_{2}-\mathrm{CH}, \mathbf{H}_{3}, \mathbf{H}_{5}$ and $\mathbf{H}_{6}$ of indol-CO), 7.55-7.80 (m, $2 \mathrm{H}, \mathbf{H}_{\mathbf{4}}$ and $\mathbf{H}_{7}$ of indol-CO), 10.36 (s, 1H, CH-CO-NH-NH-CO), 10.56 (s, 1H, CO-NH-NH-CO-Ph), 11.77 (s, 1H, NH of indole); MS m/z 348.00 (5.41), 143.95 (42.20), 90.95(100); Anal. $\left(\mathrm{C}_{26} \mathrm{H}_{24} \mathrm{~N}_{4} \mathrm{O}_{4}\right) \mathrm{C}, \mathrm{H}, \mathrm{N}$.

\section{(S)-2-Benzyloxycarbonylamino-3-phenyl-N'-(3-pyridylcarbonyl)} propionohydrazide (4)

A mixture of (a) $(0.50 \mathrm{~g}, 1.67 \mathrm{mmol})$, HOBT $(0.28 \mathrm{~g}, 1.84$ $\mathrm{mmol})$, EDC (0.35 g, $1.84 \mathrm{mmol})$ and pyridyl-3-hydrazide $(0.25$ $\mathrm{g}, 1.84 \mathrm{mmol})$. A withe solid $(0.16 \mathrm{~g}, 22 \%) \mathrm{mp}$ 174.0-176.0: IR $\left(\mathrm{KBr}, \mathrm{cm}^{-1}\right) 3419(\mathrm{~s}, \mathrm{OH}), 3284(\mathrm{~s}, \mathrm{NH}), 3028$ (w, aromatic C-H), 1691 (s, carbamate $\mathrm{C}=\mathrm{O}), 1659$ (s, hydrazide $\mathrm{C}=\mathrm{O}), 745$ and 697 (s, aromatic monosubstitution); ${ }^{1} \mathrm{H}-\mathrm{NMR}\left(\mathrm{DMSO}-\mathrm{d}_{6},{ }^{\delta}\right.$ ) $2.84\left(\mathrm{t}, 1 \mathrm{H}, \mathrm{CH}_{\mathrm{A}} \mathrm{H}_{\mathrm{B}}-\mathrm{CH}, \mathrm{J}=12.3 \mathrm{~Hz}\right), 3.13\left(\mathrm{~d}, 1 \mathrm{H}, \mathrm{CH}_{\mathrm{A}} \mathbf{H}_{\mathbf{B}}-\mathrm{CH}\right.$, $\mathrm{J}=11.9 \mathrm{~Hz}), 4.31-4.52(\mathrm{~m}, 1 \mathrm{H}, \mathrm{CH}), 4.95\left(\mathrm{~s}, 2 \mathrm{H}, \mathrm{CH}_{2}-\mathrm{O}\right), 7.12-$ $7.44\left(\mathrm{~m}, 10 \mathrm{H}, \mathrm{C}_{6} \mathbf{H}_{5}-\mathrm{CH}_{2}-\mathrm{CH}\right.$ and $\left.\mathrm{C}_{6} \mathbf{H}_{5}-\mathrm{CH}_{2}-\mathrm{O}\right), 7.53-7.59(\mathrm{~m}$, $1 \mathrm{H}, \mathbf{H}_{5}$ of pyridyl-CO), $7.71\left(\mathrm{~d}, 1 \mathrm{H}, \mathbf{H}_{\mathbf{6}}\right.$ of pyridyl-CO, J=8.6 
$\mathrm{Hz}$ ), 8.25 (d, 1H, NH-CH, J=7.9 Hz), 8.77 (d, 1H, $\mathbf{H}_{4}$ of pyridyl-CO, J=3.8 Hz), $9.06\left(\mathrm{~s}, 1 \mathrm{H}, \mathbf{H}_{2}\right.$ of pyridyl-CO), 10.39 (s, 1H, CH-CO-NH-NH-CO), 10.72 (s, 1H, CO-NH-NH-COpyridine); $\mathrm{MS} m / z 418.05\left(7.39, \mathrm{M}^{+}\right), 254.15$ (9.12), 91.00 (100); Anal. $\left(\mathrm{C}_{23} \mathrm{H}_{22} \mathrm{~N}_{4} \mathrm{O}_{4}\right) \mathrm{C}, \mathrm{H}, \mathrm{N}$.

(S)-2-Benzyloxycarbonylamino-N'-(3-indolylacethyl)-3-phenylpropionohydrazide (5)

From (a) $(0.40 \mathrm{~g}, 1.34 \mathrm{mmol})$, HOBT $(0.23 \mathrm{~g}, 1.47 \mathrm{mmol})$, EDC $(0.28 \mathrm{~g}, 1.47 \mathrm{mmol})$ and 3-indolylacetohydrazide $(0.28 \mathrm{~g}$, $1.47 \mathrm{mmol})$. A white solid $(0.39 \mathrm{~g}, 57 \%) \mathrm{mp}$ 156.7-158.5: IR $\left(\mathrm{KBr}, \mathrm{cm}^{-1}\right) 3279$ and $3230(\mathrm{vs}, \mathrm{N}-\mathrm{H}), 3055$ (m, aromatic CH), 2920 (w, aliphatic C-H), 1697 (s, carbamate C=O), 1667 and 1608 (vs, hydrazide $\mathrm{C}=\mathrm{O}$ ), 747 and 697 (s, aromatic monosubstitution); ${ }^{1} \mathrm{H}-\mathrm{NMR}$ (DMSO-d $\left.{ }_{6},{ }^{\delta}\right) 2.80\left(\mathrm{t}, 1 \mathrm{H}, \mathrm{CH}_{\mathrm{A}} \mathrm{H}_{\mathrm{B}^{-}}\right.$ $\mathrm{CH}, \mathrm{J}=12.2 \mathrm{~Hz}$ ), $3.05\left(\mathrm{~d}, 1 \mathrm{H}, \mathrm{CH}_{\mathrm{A}} \mathbf{H}_{\mathrm{B}}-\mathrm{CH}, \mathrm{J}=12.5 \mathrm{~Hz}\right), 3.93$ (s, 2H, CO-CH2-indole), 4.31-4.52 (m, 1H, CH), 4.95 (s, 2H, CH $\mathbf{H}_{2}-$ $\mathrm{O})$, 6.95-7.20 (m, 3H, $\mathbf{H}_{2}, \mathbf{H}_{5}$ and $\mathbf{H}_{6}$ of indolyl- $\left.\mathrm{CH}_{2}\right), 7.20-$ $7.45\left(\mathrm{~m}, 10 \mathrm{H}, \mathrm{C}_{6} \mathbf{H}_{5}-\mathrm{CH}_{2}-\mathrm{CH}\right.$ and $\left.\mathrm{C}_{6} \mathbf{H}_{5}-\mathrm{CH}_{2}-\mathrm{O}\right), 7.50-7.70(\mathrm{~m}$, $2 \mathrm{H}, \mathbf{H}_{4}$ and $\mathbf{H}_{7}$ of indolyl- $\left.\mathrm{CH}_{2}\right), 10.41$ (s, 2H, CO-NH-NH$\mathrm{CO}), 11.17\left(\mathrm{~s}, 1 \mathrm{H}, \mathrm{NH}\right.$ of indolyl- $\left.\mathrm{CH}_{2}\right) ; \mathrm{MS} \mathrm{m} / \mathrm{z} 392.05$ (11.81), 157.15 (86.51), 130.15 (100), 108.15 (48.18); Anal. $\left(\mathrm{C}_{27} \mathrm{H}_{26} \mathrm{~N}_{4} \mathrm{O}_{4}\right) \mathrm{C}, \mathrm{H}, \mathrm{N}$.

(S)- 2-Benzyloxycarbonylamino-3-phenyl-N-(3-pyrazolyl)propionamide (6)

From (a) $(0.50 \mathrm{~g}, 1.67 \mathrm{mmol})$, HOBT $(0.28 \mathrm{~g}, 1.84 \mathrm{mmol})$, EDC $(0.35 \mathrm{~g}, 1.84 \mathrm{mmol})$ and 3-aminopyrazole $(0.15 \mathrm{~g}, 1.84$ mmol). A white solid (0.23 g, 34\%) mp 113.3-114.9: IR (KBr, $\mathrm{cm}^{-1}$ ) 3268 (vs, N-H), 3032 (s, aromatic C-H), 2953 (s, aliphatic $\mathrm{C}-\mathrm{H}$ ), 1713 (vs, carbamate $\mathrm{C}=\mathrm{O}$ ), 1674 (vs, amide $\mathrm{C}=\mathrm{O}$ ), 738 and 697 (s, aromatic monosubstitution); ${ }^{1} \mathrm{H}-\mathrm{NMR}\left(\mathrm{CDCl}_{3},{ }^{\delta}\right)$ 3.05-3.28 (m, 2H, CH $\left.\mathbf{H}_{2}-\mathrm{CH}\right), 4.79-4.93(\mathrm{~m}, 1 \mathrm{H}, \mathrm{CH}), 5.06(\mathrm{dd}$, $\left.2 \mathrm{H}, \mathrm{CH}_{2}-\mathrm{O}, \mathrm{J}=12.4 / 28.0 \mathrm{~Hz}\right), 6.61(\mathrm{~d}, 1 \mathrm{H}, \mathrm{NH}-\mathrm{CH}, \mathrm{J}=9.5 \mathrm{~Hz})$, $6.84\left(\mathrm{~s}, 1 \mathrm{H}, \mathbf{H}_{4}\right.$ of pyrazolyl-NH), 7.15-7.36 $\left(\mathrm{m}, 11 \mathrm{H}, \mathbf{H}_{5}\right.$ of pyrazolyl-NH, $\mathrm{C}_{6} \mathbf{H}_{5}-\mathrm{CH}_{2}-\mathrm{CH}$ and $\mathrm{C}_{6} \mathbf{H}_{5}-\mathrm{CH}_{2}-\mathrm{O}$ ), 11.53 (ws, $1 \mathrm{H}, \mathrm{CH}-\mathrm{CO}-\mathrm{NH}$ ), 11.72 (ws, $1 \mathrm{H}, \mathrm{NH}$ of pyrazolyl-NH); MS $m / z 364.05\left(0.75, \mathrm{M}^{+}\right), 256.15$ (51.31), 108.00 (26.49), 91.00 (100); Anal. $\left(\mathrm{C}_{20} \mathrm{H}_{20} \mathrm{~N}_{4} \mathrm{O}_{3} \cdot 1 \_\mathrm{H}_{2} \mathrm{O}\right) \mathrm{C}, \mathrm{H}, \mathrm{N}$.

(S)-2-Benzyloxycarbonylamino-3-(4-methoxyphenyl)-N'-phenylpropionohydrazide (7)

From (b) $(0.65 \mathrm{~g}, 1.98 \mathrm{mmol})$, HOBT $(0.33 \mathrm{~g}, 2.18 \mathrm{mmol})$, EDC $(0.42 \mathrm{~g}, 2.18 \mathrm{mmol})$ and phenylhydrazine $(0.20 \mathrm{~mL}, 2.18$ mmol). A beige solid (0.62 g, 75\%) mp 183.0-185.0: IR ( $\mathrm{KBr}$, $\left.\mathrm{cm}^{-1}\right) 3299$ and $3241(\mathrm{~m}, \mathrm{~N}-\mathrm{H}), 3038$ (w, aromatic C-H), 2963 (w, aliphatic C-H), 1688 (s, carbamate $\mathrm{C}=\mathrm{O}$ ), 1657 (s, amide $\mathrm{C}=\mathrm{O}), 739$ and 694 (m, aromatic monosubstitution); ${ }^{1} \mathrm{H}-\mathrm{NMR}$ $\left(\mathrm{CDCl}_{3},{ }^{\delta}\right)$ 2.95-3.10 (m, 2H, $\left.\mathrm{CH}_{2}-\mathrm{CH}\right), 3.77$ (s, 3H, OCH $\mathbf{O H}_{3}$, 4.46-4.50 (m, 1H, CH), 5.06 (s, 2H, CH $-\mathrm{O}), 5.45$ (d, 1H, NH$\mathrm{CH}, \mathrm{J}=7.5 \mathrm{~Hz}), 5.98(\mathrm{~d}, 1 \mathrm{H}, \mathrm{NH}-\mathrm{NH}-\mathrm{Ph}, \mathrm{J}=3.2 \mathrm{~Hz}), 6.51(\mathrm{~d}$, $2 \mathrm{H}, \mathbf{H}_{\mathbf{2}}$ and $\mathbf{H}_{6}$ of $\left.\mathrm{C}_{6} \mathrm{H}_{5}-\mathrm{NH}, \mathrm{J}=7.8 \mathrm{~Hz}\right), 6.77-6.88\left(\mathrm{~m}, 3 \mathrm{H}, \mathbf{H}_{\mathbf{4}}\right.$ of $\mathrm{C}_{6} \mathrm{H}_{5}-\mathrm{NH}$ and $\mathbf{H}_{2}$ and $\mathbf{H}_{6}$ of $\left.\mathrm{C}_{6} \mathrm{H}_{4}-\mathrm{OCH}_{3}\right), 6.99-7.11(\mathrm{~m}, 4 \mathrm{H}$, $\mathbf{H}_{3}$ and $\mathbf{H}_{5}$ of $\mathrm{C}_{6} \mathrm{H}_{5}-\mathrm{NH}$ and $\mathbf{H}_{3}$ and $\mathbf{H}_{5}$ of $\left.\mathrm{C}_{6} \mathrm{H}_{4}-\mathrm{OCH}_{3}\right), 7.25$ (s, 5H, $\mathrm{C}_{6} \mathbf{H}_{5}-\mathrm{CH}_{2}$ ), 7.94 (ws, CH-CO-NH-NH); MS m/z 419.20 $\left(11.70, \mathrm{M}^{++}\right), 150.15$ (29.57), 121.15 (100), 91.05 (74.84); Anal. $\left(\mathrm{C}_{24} \mathrm{H}_{25} \mathrm{~N}_{3} \mathrm{O}_{4}\right) \mathrm{C}, \mathrm{H}, \mathrm{N}$.

(S)-N'-Benzoyl-2-benzyloxycarbonylamino-3-(4-methoxyphenyl) propionohydrazide $(\mathbf{8})$

From (b) (0.65 g, $1.98 \mathrm{mmol})$, HOBT (0.33 g, $2.18 \mathrm{mmol})$, EDC $(0.42 \mathrm{~g}, 2.18 \mathrm{mmol})$ and phenylhydrazide $(0.30 \mathrm{~g}, 2.18 \mathrm{mmol})$. A beige solid $(0.36 \mathrm{~g}, 41 \%) \mathrm{mp}$ 208.0-209.2: IR $\left(\mathrm{KBr}, \mathrm{cm}^{-1}\right)$ 3280 (s, N-H), 3033 (w, aromatic C-H), 2925 (w, aliphatic C$\mathrm{H}$ ), 1689 (vs, carbamate $\mathrm{C}=\mathrm{O}$ ), 1655 (vs, hydrazide $\mathrm{C}=\mathrm{O}$ ), 824 $(\mathrm{m}$, aromatic $p$-disubstitution), 745 and $695(\mathrm{~m}$, aromatic monosubstitution); ${ }^{1} \mathrm{H}-\mathrm{NMR}\left(\mathrm{CDCl}_{3},{ }^{\delta}\right)$ 2.90-3.12 (m, 2H, $\left.\mathrm{CH}_{2}-\mathrm{CH}\right)$, $3.68\left(\mathrm{~s}, 3 \mathrm{H}, \mathrm{OCH}_{3}\right), 4.50-4.60(\mathrm{~m}, 1 \mathrm{H}, \mathrm{CH}), 4.91-5.06(\mathrm{dd}, 2 \mathrm{H}$, $\mathrm{CH}_{2}-\mathrm{O}, \mathrm{J}=12.3 / 18.8 \mathrm{~Hz}$ ), 5.53 (ws, $\left.1 \mathrm{H}, \mathrm{NH}-\mathrm{CH}\right), 6.71$ (d, 2H,
$\mathbf{H}_{3}$ and $\mathbf{H}_{5}$ of $\left.\mathrm{C}_{6} \mathrm{H}_{4}-\mathrm{OCH}_{3}, \mathrm{~J}=8.5 \mathrm{~Hz}\right), 7.04\left(\mathrm{~d}, 2 \mathrm{H}, \mathbf{H}_{2}\right.$ and $\mathbf{H}_{6}$ de $\left.\mathrm{C}_{6} \mathrm{H}_{4}-\mathrm{OCH}_{3}, \mathrm{~J}=8.4 \mathrm{~Hz}\right), 7.10-7.25\left(\mathrm{~m}, 5 \mathrm{H}, \mathrm{C}_{6} \mathbf{H}_{5}-\mathrm{CH}_{2}-\mathrm{O}\right), 7.34$ $\left(\mathrm{t}, 2 \mathrm{H}, \mathbf{H}_{3}\right.$ and $\mathbf{H}_{5}$ of $\left.\mathrm{C}_{6} \mathrm{H}_{5}-\mathrm{CO}, \mathrm{J}=7.1 \mathrm{~Hz}\right), 7.46\left(\mathrm{t}, 1 \mathrm{H}, \mathbf{H}_{4}\right.$ of $\left.\mathrm{C}_{6} \mathrm{H}_{5}-\mathrm{CO}, \mathrm{J}=7.3 \mathrm{~Hz}\right), 7.73\left(\mathrm{~d}, 2 \mathrm{H}, \mathbf{H}_{2}\right.$ and $\mathbf{H}_{6}$ of $\mathrm{C}_{6} \mathrm{H}_{5}-\mathrm{CO}, \mathrm{J}=7.5$ $\mathrm{Hz}$ ), 8.90-9.30 (ws, 2H, CO-NH-NH-CO); MS m/z 339.05 (4.01), 150.15 (1.69), 121.15 (100), 115.00 (40.58), 91.05 (63.37); Anal. $\left(\mathrm{C}_{25} \mathrm{H}_{25} \mathrm{~N}_{3} \mathrm{O}_{3}\right) \mathrm{C}, \mathrm{H}, \mathrm{N}$.

(S)-2-Benzyloxycarbonylamino-N'-(2-indolylcarbonyl)-3-(4methoxyphenyl)propionohydrazide (9)

From (b) $(0.30 \mathrm{~g}, 0.91 \mathrm{mmol})$, HOBT $(0.16 \mathrm{~g}, 1.04 \mathrm{mmol})$, EDC $(0.20 \mathrm{~g}, 1.04 \mathrm{mmol})$ and indol-2-hydrazide $(0.19 \mathrm{~g}, 1.04$ $\mathrm{mmol})$. A beige solid $(0.20 \mathrm{~g}, 48 \%) \mathrm{mp}$ 185.5-186.5: IR (KBr, $\left.\mathrm{cm}^{-1}\right) 3359$ (vs, N-H), 3038 (w, aromatic C-H), 2963 (w, aliphatic C-H), 1707 (vs, carbamate $\mathrm{C}=\mathrm{O}$ ), 1654 and 1606 (vs, hydrazide $\mathrm{C}=\mathrm{O}$ ), 822 (s, aromatic $p$-disubstitution), 743 (vs, aromatic monosubstitution); ${ }^{1} \mathrm{H}-\mathrm{NMR}$ (DMSO-d ${ }_{6},{ }^{\delta}$ ) 2.81 $\left(\mathrm{t}, 1 \mathrm{H}, \mathrm{CH}_{\mathrm{A}} \mathrm{H}_{\mathrm{B}}-\mathrm{CH}, \mathrm{J}=12.2 \mathrm{~Hz}\right), 3.11\left(\mathrm{~d}, 1 \mathrm{H} \mathrm{CH} \mathrm{CH}_{\mathrm{A}} \mathbf{H}_{\mathbf{B}}-\mathrm{CH}\right.$, $\mathrm{J}=13.7 \mathrm{~Hz}$ ), 3.74 (s, 3H, OCH $\left.\mathbf{H}_{3}\right), 4.38$ (ws, 1H, CH), 4.97 (s, $\left.2 \mathrm{H}, \mathrm{CH}_{2}-\mathrm{O}\right), 6.87\left(\mathrm{~d}, 2 \mathrm{H}, \mathbf{H}_{3}\right.$ and $\mathbf{H}_{5}$ of $\mathrm{C}_{6} \mathrm{H}_{4}-\mathrm{OCH}_{3}, \mathrm{~J}=8.2$ $\mathrm{Hz}), 7.07\left(\mathrm{t}, 1 \mathrm{H}, \mathbf{H}_{5}\right.$ of indol-CO, J=7.2 Hz), 7.10-7.40 (m, $9 \mathrm{H}, \mathrm{C}_{6} \mathbf{H}_{5}-\mathrm{CH}_{2}, \mathbf{H}_{2}$ and $\mathbf{H}_{6}$ of $\mathrm{C}_{6} \mathrm{H}_{4}-\mathrm{OCH}_{3}$ and $\mathbf{H}_{6}$ and $\mathbf{H}_{3}$ of indol-CO), $7.47\left(\mathrm{~d}, 1 \mathrm{H}, \mathbf{H}_{4}\right.$ of indol- $\left.\mathrm{CO}, \mathrm{J}=8.0 \mathrm{~Hz}\right), 7.66(\mathrm{~d}$, $1 \mathrm{H}, \mathbf{H}_{7}$ of indol-CO, J=8.4 Hz), $7.98(\mathrm{~d}, 1 \mathrm{H}, \mathrm{NH}-\mathrm{CH}, \mathrm{J}=8.3$ $\mathrm{Hz}), 10.31$ (s, 1H, CH-CO-NH-NH-CO), 10.52 (s, 1H, CONH-NH-CO-indole), 11.74 (s, 1H, NH of indol-CO); MS m/ z 335.15 (7.99), 144.05 (55.08), 121.05 (100); Anal. $\left(\mathrm{C}_{27} \mathrm{H}_{26} \mathrm{~N}_{4} \mathrm{O}_{5}\right) \mathrm{C}, \mathrm{H}, \mathrm{N}$.

(S)-2-Benzyloxycarbonylamino-3-(4-methoxyphenyl)-N'-(3pyridylcarbonyl)propionohydrazide $(\mathbf{1 0})$

From (b) (0.30 g, $0.91 \mathrm{mmol})$, HOBT (0.15 g, $0.99 \mathrm{mmol})$, EDC (0.19 g, $0.99 \mathrm{mmol})$ and pyridyl-3-hydrazide (0.14 g, 0.99 mmol). A white solid (0.25 g, 61\%) mp 184.0-186.0: IR (KBr, $\left.\mathrm{cm}^{-1}\right) 3279(\mathrm{~s}, \mathrm{~N}-\mathrm{H}), 3033(\mathrm{w}$, aromatic C-H), $2958(\mathrm{w}$, aliphatic $\mathrm{C}-\mathrm{H}$ ), 1691 ( $\mathrm{s}$, carbamate $\mathrm{C}=\mathrm{O}$ ), 1660 (s, hydrazide $\mathrm{C}=\mathrm{O}$ ), 813 (w, aromatic p-disusbtitution), 751 and $704(\mathrm{~m}$, aromatic monosubstitution); ${ }^{1} \mathrm{H}-\mathrm{NMR}\left(\mathrm{CDCl}_{3},{ }^{\delta}\right)$ 2.93-3.16 (m, $\left.2 \mathrm{H}, \mathrm{CH}_{2}-\mathrm{CH}\right), 3.72\left(\mathrm{~s}, 3 \mathrm{H}, \mathrm{OCH}_{3}\right), 4.55-4.69(\mathrm{~m}, 1 \mathrm{H}, \mathrm{CH})$, 4.95-5.09 (dd, 2H, CH $2-\mathrm{O}, \mathrm{J}=12.3 / 17.3 \mathrm{~Hz}$ ), 5.66 (ws, $1 \mathrm{H}, \mathrm{NH}-$ $\mathrm{CH}), 6.76\left(\mathrm{~d}, 2 \mathrm{H}, \mathbf{H}_{3}\right.$ and $\mathbf{H}_{5}$ of $\left.\mathrm{C}_{6} \mathrm{H}_{4}-\mathrm{OCH}_{3}, \mathrm{~J}=8.4 \mathrm{~Hz}\right), 7.08$ $\left(\mathrm{d}, 2 \mathrm{H}, \mathbf{H}_{2}\right.$ and $\mathbf{H}_{6}$ of $\left.\mathrm{C}_{6} \mathrm{H}_{4}-\mathrm{OCH}_{3}, \mathrm{~J}=8.2 \mathrm{~Hz}\right), 7.20-7.36(\mathrm{~m}$, $6 \mathrm{H}, \mathrm{C}_{6} \mathbf{H}_{5}-\mathrm{CH}_{2}$ and $\mathbf{H}_{5}$ of pyridyl-CO), $8.07\left(\mathrm{~d}, 1 \mathrm{H}, \mathbf{H}_{6}\right.$ of pyridyl-CO, J=7.9 Hz), $8.70\left(\mathrm{~d}, 1 \mathrm{H}, \mathbf{H}_{\mathbf{4}}\right.$ of pyridyl-CO, J=4.8 $\mathrm{Hz}$ ), 9.01 (s, $1 \mathrm{H}, \mathbf{H}_{2}$ of pyridyl-CO), 9.37 (ws, 2H, CH-CONH-NH-CO-pyridine); MS m/z 297.15 (6.82), 121.05 (100), 91.15 (49.17); Anal. $\left(\mathrm{C}_{24} \mathrm{H}_{24} \mathrm{~N}_{4} \mathrm{O}_{5}\right) \mathrm{C}, \mathrm{H}, \mathrm{N}$.

(S)-2-Benzyloxycarbonylamino-3-(4-methoxyphenyl)-N'phenylsulphonylpropionohydrazide (11)

From (b) $(0.30 \mathrm{~g}, 0.91 \mathrm{mmol})$, HOBT $(0.15 \mathrm{~g}, 0.99 \mathrm{mmol})$, EDC $(0.19 \mathrm{~g}, 0.99 \mathrm{mmol})$ and benzenesulfonyl hydrazide $(0.17$ $\mathrm{g}, 0.99 \mathrm{mmol})$. A beige solid $(0.17 \mathrm{~g}, 38 \%) \mathrm{mp} 167.0-169.0$ : IR $\left(\mathrm{KBr}, \mathrm{cm}^{-1}\right) 3303(\mathrm{~s}, \mathrm{~N}-\mathrm{H}), 3063(\mathrm{w}$, aromatic C-H), 2962 (w, aliphatic C-H), 1691 (s, carbamate $\mathrm{C}=\mathrm{O}$ ), 1653 (s, hydrazide $\mathrm{C}=\mathrm{O}), 1360$ and $1173\left(\mathrm{~s}, \mathrm{SO}_{2}\right), 834(\mathrm{~m}$, aromatic $p$ disubstitution), 734 and $689(\mathrm{~m}$, aromatic monosubstitution); ${ }^{1} \mathrm{H}-\mathrm{NMR}\left(\mathrm{CDCl}_{3},{ }^{\delta}\right)$ 2.56-2.70 (m, 2H, CH $\left.\mathbf{H}_{2}-\mathrm{CH}\right), 3.69(\mathrm{~s}, 3 \mathrm{H}$, $\left.\mathrm{OCH}_{3}\right), 4.19-4.27(\mathrm{~m}, 1 \mathrm{H}, \mathrm{CH}), 5.00$ (s, 2H, CH $\left.\mathbf{H}_{2}-\mathrm{O}\right), 6.69$ (d, $2 \mathrm{H}, \mathbf{H}_{3}$ and $\mathbf{H}_{5}$ of $\left.\mathrm{C}_{6} \mathrm{H}_{4}-\mathrm{OCH}_{3}, \mathrm{~J}=8.5 \mathrm{~Hz}\right), 6.86\left(\mathrm{~d}, 2 \mathrm{H}, \mathbf{H}_{2}\right.$ and $\mathbf{H}_{6}$ of $\left.\mathrm{C}_{6} \mathrm{H}_{4}-\mathrm{OCH}_{3}, \mathrm{~J}=8.5 \mathrm{~Hz}\right), 7.19-7.53\left(\mathrm{~m}, 9 \mathrm{H}, \mathrm{C}_{6} \mathbf{H}_{5}-\mathrm{CH}_{2}\right.$ and $\mathbf{H}_{2}, \mathbf{H}_{3}, \mathbf{H}_{5}$ and $\mathbf{H}_{6}$ of $\left.\mathrm{C}_{6} \mathrm{H}_{5}-\mathrm{SO}_{2}\right), 7.79\left(\mathrm{~d}, 1 \mathrm{H}, \mathbf{H}_{4}\right.$ of $\mathrm{C}_{6} \mathrm{H}_{5^{-}}$ $\mathrm{SO}_{2}, \mathrm{~J}=7.4 \mathrm{~Hz}$ ), 9.05 (ws, 2H, CO-NH-NH-SO ${ }_{2}$; MS $\mathrm{m} / z$ 332.15 (7.19), 284.20 (1.36), 121.05 (100), 91.15 (94.19); Anal. $\left(\mathrm{C}_{24} \mathrm{H}_{25} \mathrm{~N}_{3} \mathrm{SO}_{6}\right) \mathrm{C}, \mathrm{H}, \mathrm{N}$.

(S)-2-Benzyloxycarbonylamino-N'-(3-indolylacethyl)-3-(4methoxyphenyl)propionohydrazide (12)

From (b) $(0.57 \mathrm{~g}, 1.76 \mathrm{mmol})$, HOBT $(0.30 \mathrm{~g}, 1.94 \mathrm{mmol})$, 
EDC (0.37 g, $1.94 \mathrm{mmol})$ and 3-indolylacetohydrazide $(0.37 \mathrm{~g}$, $1.94 \mathrm{mmol})$. A beige solid $(0.51 \mathrm{~g}, 53 \%) \mathrm{mp} \mathrm{194.0-195.5:} \mathrm{IR}$ $\left(\mathrm{KBr}, \mathrm{cm}^{-1}\right) 3410$ and $3272(\mathrm{vs}, \mathrm{N}-\mathrm{H}), 3034$ (s, aromatic CH), 2949 (m, aliphatic C-H), 1703 (vs, carbamate C=O), 1602 (vs, hydrazide $\mathrm{C}=\mathrm{O}$ ), 824 ( $\mathrm{s}$, aromatic $p$-disubstitution), 735 and 698 (vs, aromatic monosubstitution); ${ }^{1} \mathrm{H}-\mathrm{NMR}$ (DMSO$\left.\mathrm{d}_{6},{ }^{\delta}\right) 2.73\left(\mathrm{t}, 1 \mathrm{H}, \mathrm{CH}_{\mathrm{A}} \mathrm{H}_{\mathrm{B}}-\mathrm{CH}, \mathrm{J}=12.0 \mathrm{~Hz}\right), 2.98(\mathrm{~d}, 1 \mathrm{H}$ $\mathrm{CH}_{\mathrm{A}} \mathbf{H}_{\mathbf{B}}-\mathrm{CH}, \mathrm{J}=12.3 \mathrm{~Hz}$ ), $3.61\left(\mathrm{~s}, 2 \mathrm{H}, \mathrm{CH}_{2}\right.$-indole), $3.71(\mathrm{~s}$, $\left.3 \mathrm{H}, \mathrm{OCH}_{3}\right), 4.30$ (ws, $\left.1 \mathrm{H}, \mathrm{CH}\right), 4.95\left(\mathrm{~s}, 2 \mathrm{H}, \mathrm{C}_{6} \mathrm{H}_{5}-\mathrm{CH}_{2}-\mathrm{O}\right.$ ), $6.84\left(\mathrm{~d}, 2 \mathrm{H}, \mathbf{H}_{3}\right.$ and $\mathbf{H}_{5}$ of $\left.\mathrm{C}_{6} \mathrm{H}_{4}-\mathrm{OCH}_{3}, \mathrm{~J}=7.3 \mathrm{~Hz}\right), 6.90-7.40$ (ws, $11 \mathrm{H}, \mathrm{C}_{6} \mathbf{H}_{5}-\mathrm{CH}_{2}-\mathrm{O}, \mathbf{H}_{2}$ and $\mathbf{H}_{6}$ of $\mathrm{C}_{6} \mathrm{H}_{4}-\mathrm{OCH}_{3}, \mathbf{H}_{2}, \mathbf{H}_{5}$ and $\mathbf{H}_{6}$ of indolyl- $\mathrm{CH}_{2}$ and $\left.\mathrm{NH}-\mathrm{CH}\right), 7.55-7.75\left(\mathrm{~m}, 2 \mathrm{H}, \mathbf{H}_{4}\right.$ and $\mathbf{H}_{7}$ of indolyl- $\mathrm{CH}_{2}$ ), 10.17 (s, 2H, CO-NH-NH-CO), 10.92 (s, $1 \mathrm{H}, \mathrm{NH}$ of indolyl- $\left.\mathrm{CH}_{2}\right) ; \mathrm{MS} \mathrm{m} / \mathrm{z} 500.20\left(2.42, \mathrm{M}^{+\cdot}\right)$, 392.05 (4.80), 157.15 (100), 130.15 (77.01), 108.15 (68.11); Anal. $\left(\mathrm{C}_{28} \mathrm{H}_{28} \mathrm{~N}_{4} \mathrm{O}_{5}\right) \mathrm{C}, \mathrm{H}, \mathrm{N}$.

(S)-2-Benzyloxycarbonylamino-3-(4-methoxyphenyl)-N-(3pyrazolyl)propionamide (13)

From (b) (0.50 g, $1.52 \mathrm{mmol})$, HOBT (0.26 g, $1.67 \mathrm{mmol})$, EDC (0.32 g, $1.67 \mathrm{mmol})$ and 3-aminopyrazole $(0.14 \mathrm{~g}, 1.67$ mmol). A white solid (0.28 g, 39\%) mp 167.7-169.2: IR (KBr, $\left.\mathrm{cm}^{-1}\right) 3268(\mathrm{vs}, \mathrm{N}-\mathrm{H}), 3032(\mathrm{~s}$, aromatic C-H), $2953(\mathrm{~s}$, aliphatic C-H), 1713 (vs, carbamate $\mathrm{C}=\mathrm{O}$ ), 1674 (vs, amide $\mathrm{C}=\mathrm{O}), 813(\mathrm{~m}$, aromatic $p$-disubstitution), 738 and $697(\mathrm{~s}$, aromatic monosubstitution); ${ }^{1} \mathrm{H}-\mathrm{NMR}\left(\mathrm{CDCl}_{3},{ }^{\delta}\right)$ 2.90-3.20 $\left(\mathrm{m}, 2 \mathrm{H}, \mathrm{CH}_{2}-\mathrm{CH}\right), 3.69\left(\mathrm{~s}, 3 \mathrm{H}, \mathrm{OCH}_{3}\right), 4.65-4.85(\mathrm{~m}, 1 \mathrm{H}$, CH), 4.90-5.20 (m, 2H, $\left.\mathrm{C}_{6} \mathrm{H}_{5}-\mathrm{CH}_{2}\right), 6.63-6.81\left(\mathrm{~m}, 4 \mathrm{H}, \mathbf{H}_{4}\right.$ of pyrazolyl-NH, $\mathbf{H}_{3}$ and $\mathbf{H}_{5}$ of $\mathrm{C}_{6} \mathrm{H}_{4}-\mathrm{OCH}_{3}$ and $\left.\mathrm{NH}-\mathrm{CH}\right), 7.06$ $\left(\mathrm{d}, 2 \mathrm{H}, \mathbf{H}_{2}\right.$ and $\mathbf{H}_{6}$ of $\left.\mathrm{C}_{6} \mathrm{H}_{4}-\mathrm{OCH}_{3}, \mathrm{~J}=7.8 \mathrm{~Hz}\right), 7.15-7.35(\mathrm{~m}$, $6 \mathrm{H}, \mathbf{H}_{5}$ of pyrazolyl- $\mathrm{NH}$ and $\mathrm{C}_{6} \mathbf{H}_{5}-\mathrm{CH}_{2}$ ), 11.50 (ws, $1 \mathrm{H}, \mathrm{CH}-$ CO-NH), 11.65 (ws, $1 \mathrm{H}, \mathrm{NH}$ of pyrazolyl-NH); MS $\mathrm{m} / \mathrm{z}$ 286.15 (16.41), 121.15 (100), 108.15 (49.21); Anal. $\left(\mathrm{C}_{21} \mathrm{H}_{22} \mathrm{~N}_{4} \mathrm{O}_{4}\right) \mathrm{C}, \mathrm{H}, \mathrm{N}$.

\section{PHARMACOLOGY}

\section{Binding assays}

\section{Y1 binding assay}

Binding of iodinated peptide YY (NEN) was carried out as described by Serradeil-Le $\mathrm{Gal}^{10}$ and modified as follows: incubations were performed at $30^{\circ} \mathrm{C}$ for $90 \mathrm{~min}$. with various competitor concentrations in Buffer A (Herpes/ $\mathrm{NaOH} 20 \mathrm{mM}$, $\mathrm{MgSO}_{4} 0.81 \mathrm{mM}, \mathrm{KH}_{2} \mathrm{PO}_{4} 220 \mathrm{mM}, \mathrm{CaCl}_{2} 1.26^{\mu} \mathrm{M}, \mathrm{MgSO}_{4}$ $0.81 \mathrm{mM}$ and bovine serum albumin $0.1 \%$ ) with SK-N-MC cell membranes $\left(50{ }^{\mu} \mathrm{g}\right.$ of protein $/ \mathrm{mL}$ of assay) in a total volume of $500^{\mu} 1$. Non-specific binding was determined in the presence of $1{ }^{\mu} \mathrm{M}$ NPY. The reaction was then stopped by filtration, the filters $(\mathrm{GF} / \mathrm{B}$, Whatman, pre-coated in $0.3 \% \mathrm{PEI})$ were extensively washed with buffer $\mathrm{A}$, and counted in a gamma counter (Packard).

\section{$Y 2$ binding assay}

Binding of iodinated peptide YY was conducted as described by Fuhlendorff et al. ${ }^{11}$ on membrane prepared from KANTS cells.

\section{Y5 binding assay}

Binding of iodinated peptide YY (NEN) was carried out as described by Hu et al. ${ }^{12}$. In brief, COS cells transfected with the human Y5 NPY receptor were lysed and the membranes prepared by differential centrifugation. These membranes contained about 2 pmol per $\mathrm{mg}$ of protein of this receptor. Incubations were performed in $500{ }^{\mu_{1}}$ comprising, $20 \mathrm{pM}$ final of $\left[{ }^{125} \mathrm{I}\right] \mathrm{PYY}$ in 50 ${ }^{\mu} 1.400^{\mu} 1$ of membrane suspension $(0.15 \mathrm{mg} / \mathrm{mL})$ and competitor dilutions in $50^{\mu} 1$, at $30^{\circ} \mathrm{C}$ for $2 \mathrm{~h}$. The reaction was stopped by filtration through GF/C filters (Whatman).

Note: Given the nature of the experiment the obtained values are within an acceptable variability range for this type of experiments, and therefore the standard errors are not included in the report.

\section{RESULTS AND DISCUSSION}

The carbamate derivatives were obtained as shown in Scheme 1. Formation of the carbamate is carried out by reaction of the benzyl chloroformate with the appropriate amino acid dissolved in a solution of $\mathrm{NaOH} 4 \mathrm{M}$. The use of strong basic mediums is necessary in order to solubilize the amino acids. In the reactions of the acid group of the amino acid fragment with amines, hydrazines or hydrazides, the work was carried out with $\mathrm{N}$-(3-dimethylaminopropyl)- $\mathrm{N}^{\prime}$-ethylcarbodiimide hydrochloride (EDC) and 1-hydroxybenzotriazole (HOBT), and with dichloromethane as the solvent of low dielectric constant. In each case, the corresponding amides or hydrazides were obtained. The reaction of the amino group of the amino acid does not lead to inversion of the configuration, but the reaction of the carboxyl group may give rise to different percentages of racemization. In order to avoid this, the reaction has been carried out with coupling agents that avoid racemization according to studies carried out by other authors ${ }^{13}$.

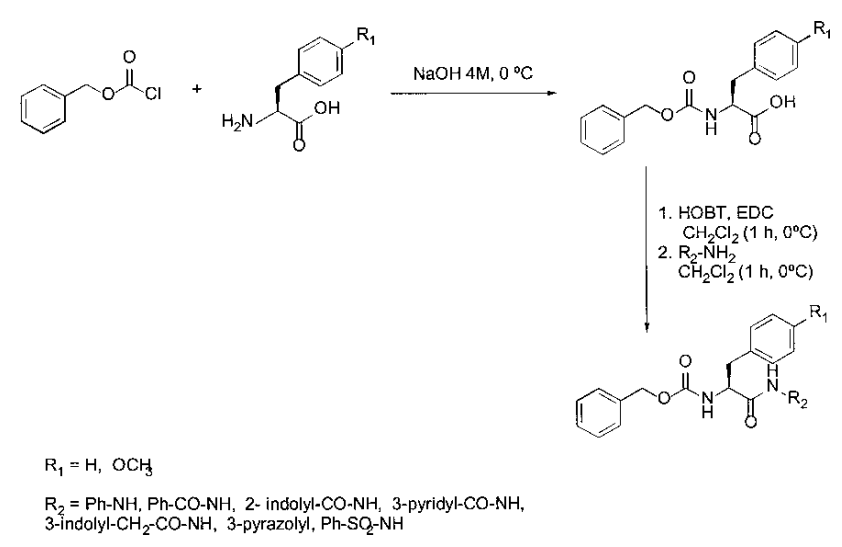

Scheme 1. Synthesis of hydrazide derivatives

The activity of compounds was evaluated by binding assay conducted as described by Félétou et al. ${ }^{14}$. Initially, the antagonist activity of the compounds was evaluated in a binding assay for Y1, Y2 and Y5 subtypes receptors. Competetive binding assays were performed using [ $\left.{ }^{125} \mathrm{I}\right]-\mathrm{PPY}$ as the radioligand, at concentrations which varied from 15 to $65 \mathrm{pM}$. The nonspecific fraction is measured in the presence of a concentration of $1{ }^{\mu} \mathrm{M}$ of NPY. The cells are incubated during a time which varied from 1 to 2 hours depending upon the classes, and the radioactivity is collected after filtration with a GF/C filter treated with PEI $0.1 \%$, before being measured. The data corresponding to the products evaluated are shown in Table 1.

The results of affinity by the NPY subtype receptors Y1, Y2, Y5, from which the antagonist activity of the synthetized molecules has been determined, indicate that the molecules which were prepared from L-phenylalanine have better behavior than those prepared from L-methyltirosine. The compound 5 $\left(\mathrm{IC}_{50}=4^{\mu} \mathrm{M}\right)$, which has a heterocyclic system of indole manifests an important level of activity. These initial results have convinced us to continue our work in the optimization of the starting base structure, searching for new highly selective nonpeptide antagonist of NPY Y5 receptors. 
Table 1. Activity of hydrazide derivatives at the Y1, Y2, and Y5 NPY receptors.

\begin{tabular}{|c|c|c|c|c|c|c|}
\hline \multirow[t]{2}{*}{ Compd. } & \multirow[t]{2}{*}{$\mathrm{R}_{1}$} & \multirow[t]{2}{*}{$\mathrm{R}_{2}$} & \multirow[b]{2}{*}{$\%$ Inhib. $\mathrm{Y}_{1}$} & \multicolumn{2}{|c|}{ Biological Activity } & \multirow[b]{2}{*}{$\%$ Inhib. $\mathrm{Y}_{5}$} \\
\hline & & & & $\%$ Inhib. $\mathrm{Y}_{2}{ }^{\mathrm{b}}$ & $\%$ Inhib. $\mathrm{Y}_{2}^{\mathrm{c}}$ & \\
\hline 1 & $-\mathrm{H}$ & Ph-NH- & $A>5 \cdot 10^{-6} M$ & $A>510^{-6} \mathrm{M}$ & I & $A>5 \cdot 10^{-6} M$ \\
\hline 2 & $-\mathrm{H}$ & $\mathrm{Ph}-\mathrm{CO}-\mathrm{NH}-$ & I & $A>5 \cdot 10^{-6} \mathrm{M}$ & I & $A>5 \cdot 10^{-6} \mathrm{M}$ \\
\hline 3 & $-\mathrm{H}$ & 2-Indolyl-CO-NH- & I & & I & I \\
\hline 4 & $-\mathrm{H}$ & 3-Pyridyl-CO-NH- & I & I & & $24 \% 10^{-5} \mathrm{M}$ \\
\hline 5 & $-\mathrm{H}$ & 3-Indolyl- $\mathrm{CH}_{2}-\mathrm{CO}-\mathrm{NH}-$ & $10 \% 10^{-5} \mathrm{M}$ & & & $36 \% 10^{-5} \mathrm{M}$ \\
\hline 6 & $-\mathrm{H}$ & 3-Pyrazolyl- & I & I & & I \\
\hline 7 & $-\mathrm{OCH}_{3}$ & $\mathrm{Ph}-\mathrm{NH}-$ & $\mathrm{A}>5 \cdot 10^{-6} \mathrm{M}$ & $A>510^{-6} \mathrm{M}$ & I & I \\
\hline 8 & $-\mathrm{OCH}_{3}$ & Ph-CO-NH- & $A>5 \cdot 10^{-6} \mathrm{M}$ & I & I & I \\
\hline 9 & $-\mathrm{OCH}_{3}$ & 2-Indolyl-CO-NH- & I & $>10^{-5}$ & I & I \\
\hline 10 & $-\mathrm{OCH}_{3}$ & 3-Pyridyl-CO-NH- & I & I & & $22 \% 10^{-5} \mathrm{M}$ \\
\hline 11 & $-\mathrm{OCH}_{3}$ & $\mathrm{Ph}-\mathrm{SO}_{2}-\mathrm{NH}-$ & $23 \% 10^{-5} \mathrm{M}$ & & & I \\
\hline 12 & $-\mathrm{OCH}_{3}$ & 3-Indolyl- $\mathrm{CH}_{2}-\mathrm{CO}-\mathrm{NH}-$ & I & & & I \\
\hline 13 & $-\mathrm{OCH}_{3}$ & 3-Pyrazolyl- & I & & & I \\
\hline $\begin{array}{l}\mathrm{I}=\text { inactive } \\
\mathrm{A}=\text { agonist }\end{array}$ & & $\begin{array}{l}\text { abit saphenous vein } \\
\text { dog saphenous vein } \\
\text { at deferens vessel } \\
\text { abbit ileum }\end{array}$ & & & & \\
\hline
\end{tabular}

\section{REFERENCES}

1. Gehlert, D. R.; Proc. Soc. Exp. Biol. Med. 1998, $218,7$.

2. Blomqvist, A. G.; Herzog, H.; Trends Neurol Sci. 1997, 20, 294.

3. Sahu, A.; Sninsky, C. A.; Kalra, Sp.; Brain Res. 1997, $755,339$.

4. Zimanyi, I. A.; Fathi, Z.; Poindexter, G. S.; Curr. Pharm. Des. 1998, 4, 349.

5. Kanataki, A.; Ishihara, A.; Asahi, S.; Tanaka, T.; Ozaki, S.; Ihara, M.; Endocrinology 1996, 137, 3177.

6. Doods, H. N.; Wieland, H. A.; Engel, W.; Regul. Pept. 1996, 65, 71 .

7. Leoluca, C, et al.; J. Clin. Invest. 1998, 102, 2136.

8. Rueger, H.; Schmidlin, T.; Rigollier, P.; Yamagichi, Y.; 1997, WO97/20283-A2.
9. Hong Pheng, L.; Quirion, R.; Iyengar, S.; Fournier, A.; Regoli, D.; Eur. J. Pharmacol. 1997, 333, R3-R5.

10. Serradeil-Le Gal, C.; Valette, G.; Rouby, P. E.; Pellet, A.; Oury-Donat, F.; Brossard, G.; Lespy, L.; Marty, E.; Neliat, G.; de Cointet, P.; Maffrand, J. P.; Le Fur, G.; FEBS Lett. 1995, 362, 192.

11. Fuhlendorff, J.; Gether, U.; Aakelund, L.; Langeland-Johansen, N.; Thogersen, H.; Melberg, S.G.; Olsen, U.B.; Thatrup, O.; Schwartz, T. W.; Proc. Natl. Acad. Sci. 1990, 87, 182.

12. Hu, Y.; Bloomquist, B. T.; Cornfield, L. J.; DeCarr, L. B.; Flores-Riveros, J.; Friedman, L.; Jiang, P.; LewisHiggins, L.; Sadlowski, Y.; Schaefer, J.;Velazquez, N.; McCaleb, M. L.; J. Biol. Chem. 1996, 271, 26315.

13. Mizayawa, T.; Otomatsu, T.; Yamada, T.; Kuwata, S.; Tetrahedron Lett. 1984, 25, 771.

14. Feletou, M et al.; Regulatory Peptides 1998, 75-76, 221 